\title{
Hydration dynamics promote bacterial coexistence on rough surfaces
}

\author{
Gang Wang and Dani Or \\ Department of Environmental Systems Science, Institute of Terrestrial Ecosystems, ETH Zurich, \\ Universitaetstrasse, Zurich, Switzerland
}

\begin{abstract}
Identification of mechanisms that promote and maintain the immense microbial diversity found in soil is a central challenge for contemporary microbial ecology. Quantitative tools for systematic integration of complex biophysical and trophic processes at spatial scales, relevant for individual cell interactions, are essential for making progress. We report a modeling study of competing bacterial populations cohabiting soil surfaces subjected to highly dynamic hydration conditions. The model explicitly tracks growth, motion and life histories of individual bacterial cells on surfaces spanning dynamic aqueous networks that shape heterogeneous nutrient fields. The range of hydration conditions that confer physical advantages for rapidly growing species and support competitive exclusion is surprisingly narrow. The rapid fragmentation of soil aqueous phase under most natural conditions suppresses bacterial growth and cell dispersion, thereby balancing conditions experienced by competing populations with diverse physiological traits. In addition, hydration fluctuations intensify localized interactions that promote coexistence through disproportional effects within densely populated regions during dry periods. Consequently, bacterial population dynamics is affected well beyond responses predicted from equivalent and uniform hydration conditions. New insights on hydration dynamics could be considered in future designs of soil bioremediation activities, affect longevity of dry food products, and advance basic understanding of bacterial diversity dynamics and its role in global biogeochemical cycles.
\end{abstract}

The ISME Journal (2013) 7, 395-404; doi:10.1038/ismej.2012.115; published online 11 October 2012

Subject Category: microbial ecology and functional diversity of natural habitats

Keywords: bacterial coexistence; diffusion; hydration dynamics; motility

\section{Introduction}

Notwithstanding the vagaries of extreme environmental fluctuations affecting the harsh and nutrient poor environment, soil emerges as the most biologically active compartment of the biosphere hosting unparalleled bacterial diversity at all scales (Stotzky, 1997; Fenchel, 2002; Torsvik and Ovreas, 2002; Fierer and Jackson, 2006; Hibbing et al., 2010). Understanding bacterial diversity patterns and function, and determining biophysical processes that shape and maintain diversity represents a central challenge for contemporary microbial ecology (Fenchel, 2002; Hibbing et al., 2010; Fierer and Lennon, 2011). Curtis and Sloan (2004) have commented that this challenge is 'an immense and unexplored frontier in science of astronomical dimensions and of astonishing complexity'.

Soil bacteria inhabit complex and heterogeneous pore spaces where water and nutrient resources

Correspondence: D Or, Department of Environmental Systems Science, Institute of Terrestrial Ecosystems, ETH Zurich, Universitaetstrasse 16, 8092 Zurich, Switzerland.

E-mail: dani.or@env.ethz.ch

Received 2 April 2012; revised 8 August 2012; accepted 14 August 2012; published online 11 October 2012 essential for bacterial life may significantly vary across micrometeric spatial scales or entirely change within a single bacterial generation (Crawford et al., 2005; Mitchell and Kogure, 2006; Or et al., 2007; Banavar and Maritan, 2009). Hydration status and pore-space characteristics are critical factors shaping nutrient fields and bacterial motility, and are thus key to understanding bacterial interactions in soil and other porous media such as dry food products (Barton and Ford, 1997; Dens and Van Impe, 2000; Wilson et al., 2002; Chang and Halverson, 2003; Or et al., 2007; Chen and Jin, 2011). Although motility has long been argued as a key factor for survival in heterogeneous environments and for biodiversity maintenance (Fenchel, 2002; Reichenbach et al., 2007; Vos and Velicer, 2008), it is only recently that crucial processes regulating bacterial motility within liquid films forming on partially hydrated rough surfaces have been quantified (Dechesne et al., 2010; Wang and Or, 2010). These studies have shown that surface roughness and aqueous-phase configuration impose capillary and hydrodynamic constraints limiting bacterial motility, and defined a surprisingly narrow range of hydration conditions where motility could confer ecological advantage on rough surfaces. 
These are but preliminary steps toward development of a broader understanding of hydration effects on bacterial population interactions and species coexistence in unsaturated soil.

In addition to inherent spatial heterogeneity of complex soil pore spaces and the resulting configuration of the aqueous phase retained therein, dynamic fluctuations in hydration conditions common in most natural soils affect microhabitats, and thus greatly influence growth rates and community compositions. Such fluctuations and associated aqueous-phase reconfiguration may create new niches that may shelter less competitive communities, or restrict diffusion in support of thriving communities, thereby enhancing bacterial diversity (Torsvik and Ovreas, 2008). Like in other ecological systems, studies have shown that fluctuations in hydration conditions could lead to significant decay in bacterial biomass and alter community composition (Fierer and Schimel, 2002; Gordon et al., 2008). Nevertheless, most previous studies have focused on bacterial survival and population recovery with little consideration of the role of hydration dynamics on interactions among competing bacterial populations (Prosser et al., 2007; Torsvik and Ovreas, 2008). Not surprisingly, a mechanistic picture of how soil bacterial diversity is promoted and maintained remains sketchy (Torsvik and Ovreas, 2008; Ponciano et al., 2009). Progress in resolving mechanisms responsible for promoting or limiting bacterial competition and diversity, and the development of predictive tools require quantitative modeling capable of systematic consideration of bio-physicochemical processes and ecological interactions at appropriate spatial and temporal scales (Prosser et al., 2007; O’Donnell et al., 2007; Banavar and Maritan, 2009).

We study interactions between hydration dynamics and diffusional heterogeneity affecting bacterial growth, motility, competition and species coexistence on partially hydrated rough surfaces. We employed a hybrid model that couples individual-based description of cell growth, motion and interactions within a nutrient field described by a (continuum-based) reaction - diffusion model (Kreft et al., 1998; Dechesne et al., 2010). The model resolves spatial and temporal nutrient diffusion fields subjected to prescribed boundary conditions, heterogeneity and local nutrient interception by individual cells. In addition, the model explicitly tracks motions and life histories of all individual cells within a population considering local hydrodynamic and capillary constraints to motility (due to aqueous-phase configuration).

\section{Materials and methods}

\section{Modeling heterogeneous rough surface and water configuration}

Natural surfaces are represented as two-dimensional networks of roughness elements with different characteristics arranged on a lattice (Dechesne et al., 2010) in which bacterial populations grow, interact and compete. The roughness network captures salient aspects of aqueous-phase retention and spatial organization of real surfaces, while providing a tractable representation of physical processes such as water films, hydraulic connectivity and effective diffusion of real porous media (Blunt, 2001; Dechesne et al., 2010). The amount of aqueous phase retained within roughness element was calculated as a function of ambient matric potential value (or relative humidity in the air) and surface roughness geometry (Or et al., 2007). The connectivity of aqueous networks and the effective sizes of connected aqueous elements capable of supporting bacterial motility were deduced from roughness element geometry, and invoking universal percolation theory arguments for network fragmentation (Berkowitz and Ewing, 1998). An example of the resulting connected clusters of water-filled channels are depicted in Figures 1a and b for a roughness network under different matric potential values, highlighting increased fragmentation of aquatic habitats as the surface becomes drier.

Nutrient diffusion and bacterial motility and growth on partially hydrated rough surfaces

The effective diffusion coefficient $D_{\mathrm{S}}$ for nutrients varies with hydration conditions (higher for wetter
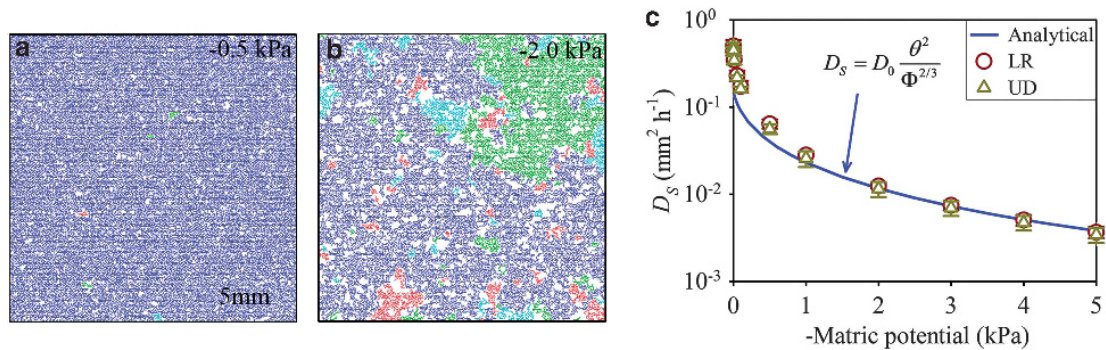

Figure 1 Aqueous configurations and nutrient diffusive flux on roughness network. Aqueous-phase configuration on a model roughness network under (a) wet and (b) dry conditions; and (c) simulated (mean \pm s.d., $n=5$ ) and analytical effective nutrient diffusion coefficients as a function of matric potential value. UD (Up to Down) and LR (Left to Right) represent simulated diffusion coefficients with flux from up to down and from left to right boundaries of the simulation domain, respectively. Colors in (a) and (b) mark different water-filled bond clusters (aqueous fragmentation) available for cell motility (with effective water film thickness larger than a typical cell size of $1 \mu \mathrm{m})$. Clusters containing bond numbers $<10$ are not plotted. 
conditions), and thus is a key parameter for determining bacterial growth rate and population carrying capacity of unsaturated surfaces. For simplicity, we expressed the relative diffusion coefficient as a function of hydration state (water content or matric potential) according to the classical Millington and Quirk model that was originally developed for soil (Moldrup et al., 2003),

$$
D_{S}=D_{0} \frac{\theta^{2}}{\Phi^{2 / 3}},
$$

where $D_{0}$ is nutrient diffusion coefficient in bulk water, $\Phi$ is effective 'porosity' of rough surface (relative to smooth surface), that is calculated according to,

$$
\Phi=\frac{2 \sqrt{3}}{3\langle H\rangle l} \int_{\Omega_{\alpha}} \int_{\Omega_{H}} H^{2} \tan \left(\frac{\alpha}{2}\right) \mathrm{d} \alpha \mathrm{d} H,
$$

where $\langle H\rangle$ is the expected value of channel/bond height (considering the effective height of the domain equals to the value of three times of the mean height of channels/bonds), $l$ is the length of a roughness element (channel/bond), $\alpha$ and $H$ are the spanning angles and height of a roughness element, with the intervals of $\Omega_{\alpha}$ and $\Omega_{H}$, respectively (Dechesne et al., 2010), and $\theta$ is the volumetric water content, which can be estimated as a function of ambient hydration status (matric potential, $\psi$ ) and surface roughness characteristics according to,

$$
\theta=\sum_{i} \theta_{i}(\psi) /(3\langle H\rangle A),
$$

where $\theta_{i}(\psi)$ is the volumetric water content of a certain roughness element at matric potential value $\psi$ (Dechesne et al., 2010), $A$ is the surface area (summation is over all elements in the network). Monte Carlo simulations of diffusive fluxes across the unsaturated roughness network yield effective nutrient diffusion coefficient similar to those obtained from macroscopic Millington and Quirk model (Moldrup et al., 2003), as depicted in Figure 1c. For high matric potential values (wet conditions) large nutrient diffusive fluxes are supported across the roughness network, with effective nutrient diffusion coefficient of up to $0.5 \mathrm{~mm}^{2} \mathrm{~h}^{-1}$ under $-0.0001 \mathrm{kPa}$ (wettest conditions considered) similar to values in the range of $0.4-0.8 \mathrm{~mm}^{2} \mathrm{~h}^{-1}$ for soils at near saturation found by Darrah (1991) and Moldrup et al. (2003). The drying of a rough surface (lower matric potential) is associated with a significant decline in effective nutrient diffusion coefficient within a few kilopascal drop in matric potential value.

Flagellated motility is the primary mode of selfpropulsion of bacterial cells in planktonian form within aqueous films (Darnton and Berg, 2008; Dechesne et al., 2010). On partially hydrated rough surfaces, flagellated motion is gradually restricted due to cell-wall viscous drag and capillary pinning forces experienced by cells within thin aqueous films. These effects are succinctly lumped into a relationship between cell size and effective water film thickness- $d(\psi)$ (which can be calculated as a function of matric potential and geometrical features of a roughness element according to $d(\psi)=R(\psi)(1-\sin (\alpha / 2)) /(1+\sin (\alpha / 2))$, with $R(\psi)$ of the radius of the liquid interfacial meniscus formed in a roughness element, see Long and Or, 2005 for more detail). We explicitly consider capillary and hydrodynamic limitations to cell motility (expressed as cell velocity, $V$ ) as a function of matric potential in the following expression:

$$
V(\psi)=V_{0} \frac{F_{M}-F_{C}(d(\psi))-F_{\lambda}(d(\psi))}{F_{M}}
$$

$\left(V(\psi)=0, \quad\right.$ while $\left.\quad F_{M}-F_{C}(d(\psi))-F_{\lambda}(d(\psi))<0\right)$, with $V_{0}$ of mean cell velocity in bulk water, and $F_{M}, F_{C}$ and $F_{\lambda}$ are the viscous drag forces opposing motion in bulk water (equal to the maximum flagellar propulsive force), the viscous force associated with cell-wall hydrodynamic interactions and the capillary pinning force, respectively (Dechesne et al., 2010). In addition, the receding air - water interfaces results in thinning of film thickness effectively, disconnecting bacterial aqueous habitats previously hydraulically connected under wet conditions and further limit bacterial motion (Or et al., 2007; Dechesne et al., 2010; Wang and Or, 2010). Therefore, cell velocity within each roughness element is determined by matric potential value and geometrical features of a roughness element. Nutrient diffusion, bacterial growth and nutrient consumption within a channel/bond are solved based on the well-established reaction diffusion model (Kreft et al., 1998; Dechesne et al., 2010).

Simulations of bacterial growth and competition on dynamically hydrated rough surfaces

The hybrid modeling framework discussed above supports a highly resolved description of spatial and temporal nutrient diffusion fields shaped by surface and aqueous-phase heterogeneity, and by local nutrient interception by individual cells. To provide a baseline for the joint effects of physicochemical heterogeneity at microscale with variations in hydration status on bacterial motility, growth and population interactions on rough surfaces, we conducted Monte Carlo simulations for various (static) hydration conditions expressed as matric potential values of $-0.5,-0.9,-2.0$ and $-3.5 \mathrm{kPa}$ (10 replicates for each matric potential value using newly generated roughness network for each replicate). The baseline static simulation results were compared with simple dynamic hydration cycles (three simulations for each hydration sequence), starting with water potential at $-2.0 \mathrm{kPa}$ that varied subsequently between -2.0 and $-0.5 \mathrm{kPa}$ at every 12 and $48 \mathrm{~h}$, respectively. 
Monte Carlo simulations were performed on replicate roughness networks, representing rough surface with physical size of $34.4 \times 34.4 \mathrm{~mm}^{2}$ (with $200 \times 173$ sites on hexagonal lattice and bond length of $0.2 \mathrm{~mm}$ ), with the parameters of network as described from Wang and Or (2010). The hybrid individual-based model simulated growth and interactions among 60 cells that were inoculated at three sites (Figure 2a) each consisting of 20 cells (10 of each competing species). The nutrient concentration across the entire simulation domain was initially constant, and subsequently we maintained constant concentrations only at the boundaries of the network throughout the simulation period. Nutrient distribution and diffusion are supported by the variable aqueous network resulted from various hydration states (Long and Or, 2005). The physiological parameters used for modeling growth, interactions and nutrient consumption of two competing bacterial species are summarized in Table 1 (Kreft et al., 1998). Note the specific growth rate for the superior species (Sp1) is higher than for the inferior species (Sp2), reflecting the sole physiological advantage of Sp1 in nutrient interception and growth rate.

The fitness of Sp2 relative to Sp1 was computed according to (Elena and Lenski, 2003),

$$
R F=\left(\frac{W_{2}}{W_{02}}\right) /\left(\frac{W_{1}}{W_{01}}\right),
$$

where $W_{02}$ and $W_{2}$ are the initial and final populations (simulated up to nutrient carrying capacity of a habitat) of Sp2, whereas $W_{01}$ and $W_{1}$ are those of Sp1.

\section{Results}

Bacterial growth and competition on rough surfaces under static hydration conditions

We first describe simulation results for surfaces under static hydration conditions as reference for simulations considering dynamic hydration status for similar mean aqueous-phase content. Figure 2 depicts patterns and population growth curves of two competing bacterial species on rough surfaces under different (but static) hydration conditions. As expected, under wet conditions (matric potential value of $-0.5 \mathrm{kPa}$ ), the total bacterial population expanded rapidly with total population size exceeding $10^{5}$ cells within $70 \mathrm{~h}$ after initial inoculation (Figure 2a). A small reduction in matric potential value (more negative, thus drier surfaces) from -0.5 to $-0.9 \mathrm{kPa}$ resulted in a $25 \%$ decrease in population size; only a few hundred cells survived with subsequent reduction to $-2.0 \mathrm{kPa}$ (Figures $2 \mathrm{~b}$ and d); and finally, no significant bacterial growth was possible for $-3.5 \mathrm{kPa}$ for similar time frames. The highest population density was found at the front of an expanding wave reflecting nutrient conditions and interception at the expanding front as seen in Figure 2a (Tsyganov and Ivanitsky, 2006; Saragosti et al., 2011). In general, the thinning of effective aqueous film thickness (Figure 2c) and fragmentation
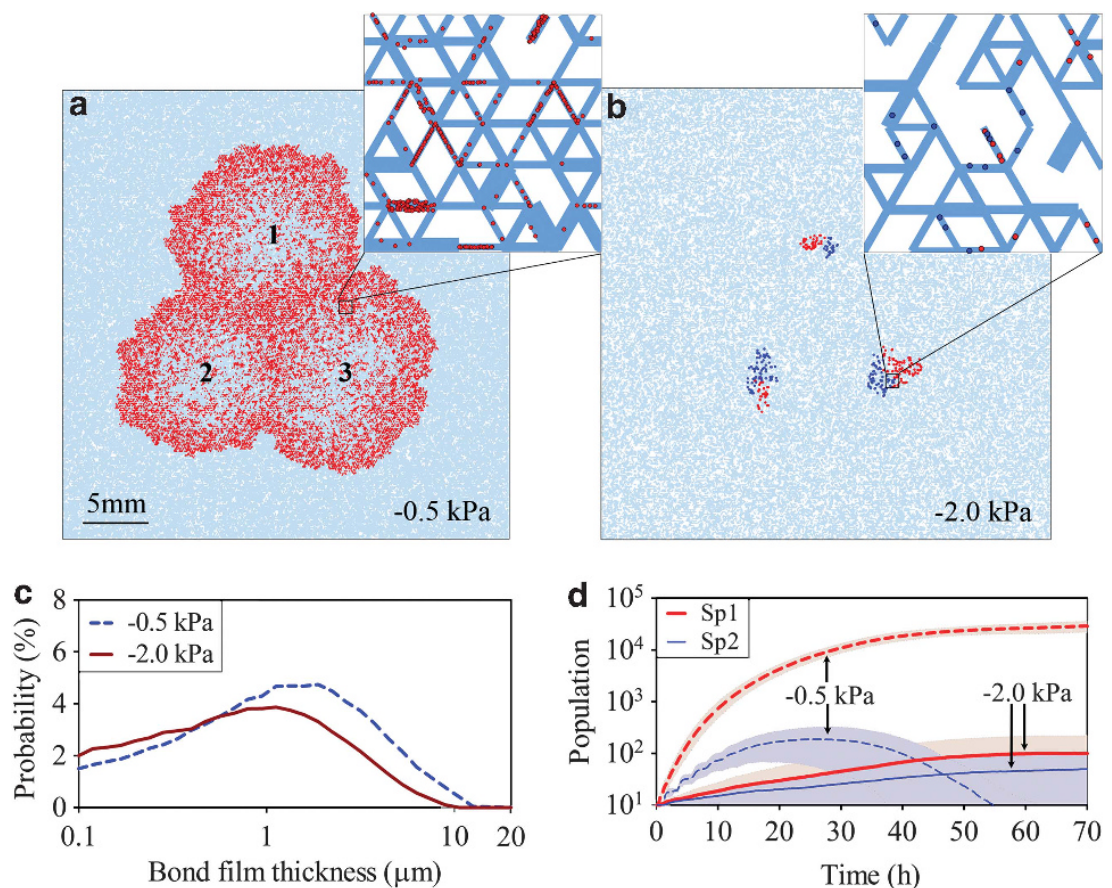

Figure 2 Bacterial growth patterns and population dynamics on rough surfaces under different static hydration conditions. Simulated bacterial growth patterns under $(\mathbf{a})$ wet $(-0.5 \mathrm{kPa})$ and $(\mathbf{b})$ dry $(-2.0 \mathrm{kPa})$ conditions, $70 \mathrm{~h}$ after inoculations; (c) water configuration (expressed as the probability density of effective bond film thickness, with film thickness $<0.1 \mu \mathrm{m}$ is not shown, see Figure 5c); and (d) bacterial population growth (mean \pm s.d., $n=30$ ) under $-0.5 \mathrm{kPa}$ (dash lines) and $-2.0 \mathrm{kPa}$ (solid lines), respectively. Red and blue spots in (a) and (b) represent individual cells of Sp1 and Sp2. Numbers in (a) mark inoculation positions. 
Table 1 Parameters describing bacterial growth and metabolism

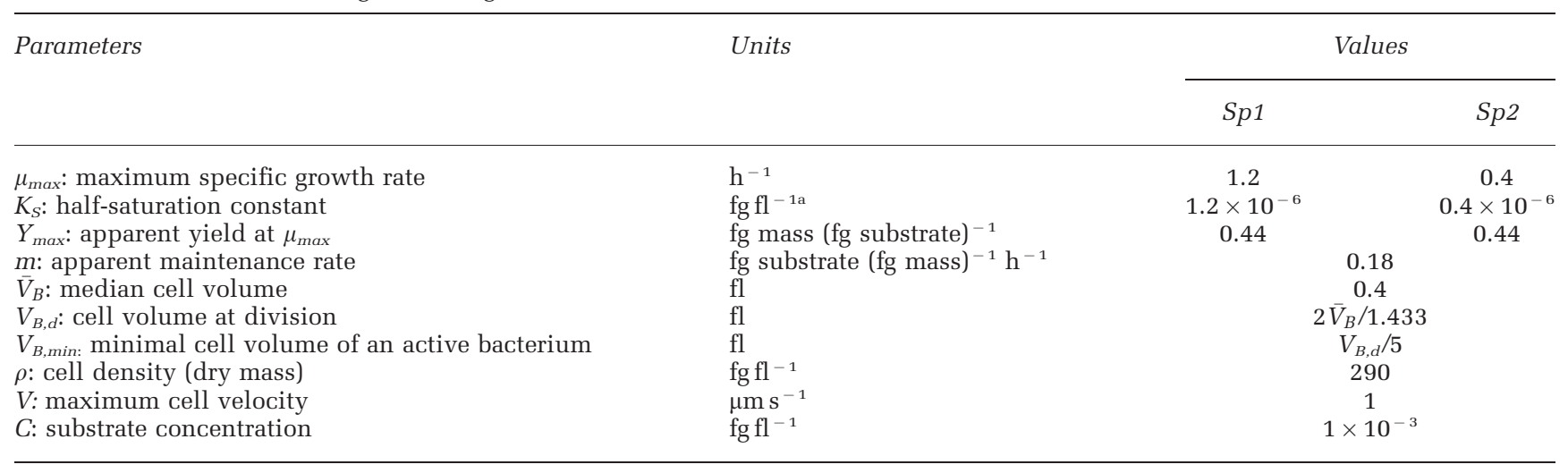

${ }^{\mathrm{a}} 1 \mathrm{fg}=1 \times 10^{-15} \mathrm{~g} ; 1 \mathrm{fl}=1 \times 10^{-15} \mathrm{l}$.

of the aqueous phase with decreasing matric potential (Figure 1b) limit nutrient diffusive fluxes (Figure 1c) and suppress cell motion and dispersion, and thus limit bacterial population growth. Simulations for static hydration conditions are in agreement with experimental observations (Drenovsky et al., 2004; Dechesne et al., 2008; Ponciano et al., 2009; Dechesne et al., 2010) and with recently published modeling results, considering a single bacterial species on unsaturated rough surfaces (Wang and Or, 2010). In addition, wet surface conditions $(-0.5 \mathrm{kPa})$ supported rapid growth by Sp1 that exhibited an exponential growth period immediately after inoculation, followed by a gradual decrease in growth rate towards a stationary phase throughout the rest of the simulation period (Figure 2d). In contrast, growth rates of Sp2 dropped rapidly, attaining negative values at $30 \mathrm{~h}$ after inoculation, leading to an eventual extinction of Sp2 (Figures 2a and d). Drier condition $(-2.0 \mathrm{kPa}$ ) suppressed growth rates for both species leading to similar population sizes at $70 \mathrm{~h}$ after inoculation with similar spatial growth patterns indicative of coexistence (Figures $2 \mathrm{~b}$ and $\mathrm{d}$ ). Further reduction in matric potential value did not affect population coexistence, albeit no significant growth of both species was simulated. Consistent with equivalent population growth sizes, comparable colony expansion rates of both species were simulated on drier rough surfaces, unlike the continuous decrease in colony expansion ratio of $\mathrm{Sp} 2$ relative $\mathrm{Sp} 1$ for simulations under wet surfaces with a matric potential value of $-0.5 \mathrm{kPa}$ (Figure 3 ).

Effects of hydration dynamics on bacterial growth and species coexistence

Our primary focus was on quantifying the role of drying and wetting cycles on bacterial population growth relative to behavior under equivalent mean (static) hydration conditions. Figure 4 depicts snapshots of simulated bacterial growth patterns and population dynamics under static and dynamic
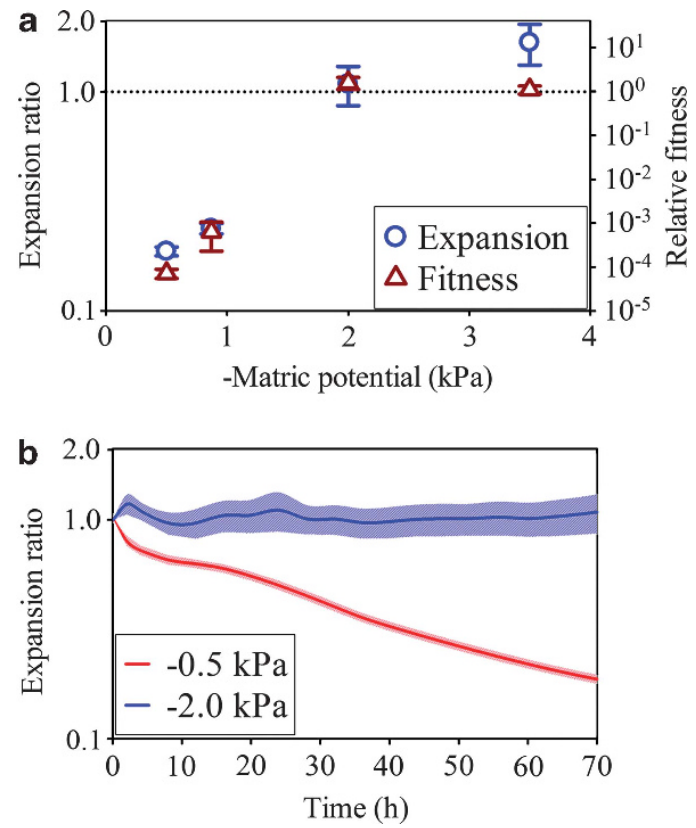

Figure 3 Bacterial colony expansion ratio and relative fitness under different static hydration conditions. (a) Simulated bacterial colony expansion ratio of $\mathrm{Sp} 2$ relative to $\mathrm{Sp} 1$, and relative fitness or Sp2 relative to $\mathrm{Sp} 1$ under various matric potential values (mean \pm s.e.m., $n=30$ ); and (b) dynamics of colony expansion ratio of $\mathrm{Sp} 2$ relative to $\mathrm{Sp} 1$ as a function of elapsed time under wet and dry hydration conditions (mean \pm s.e.m., $n=30$, shaded areas represent 1 s.e.m.).

hydration cycles (initial water potential was $-2.0 \mathrm{kPa}$, and was alternated between -2.0 and $-0.5 \mathrm{kPa}$ every 12 (short hydration cycle) and $48 \mathrm{~h}$ (long hydration cycle), respectively). Results show that the time required for similar colony sizes under short and long hydration cycles was 144 and $192 \mathrm{~h}$, respectively, as compared with $50 \mathrm{~h}$ for (equivalent) static hydration conditions. Hydration dynamics resulted in disproportional reduction in bacterial population growth during dry periods as evidenced by low-specific growth rates, which were followed by rapid recovery in population size upon rewetting 

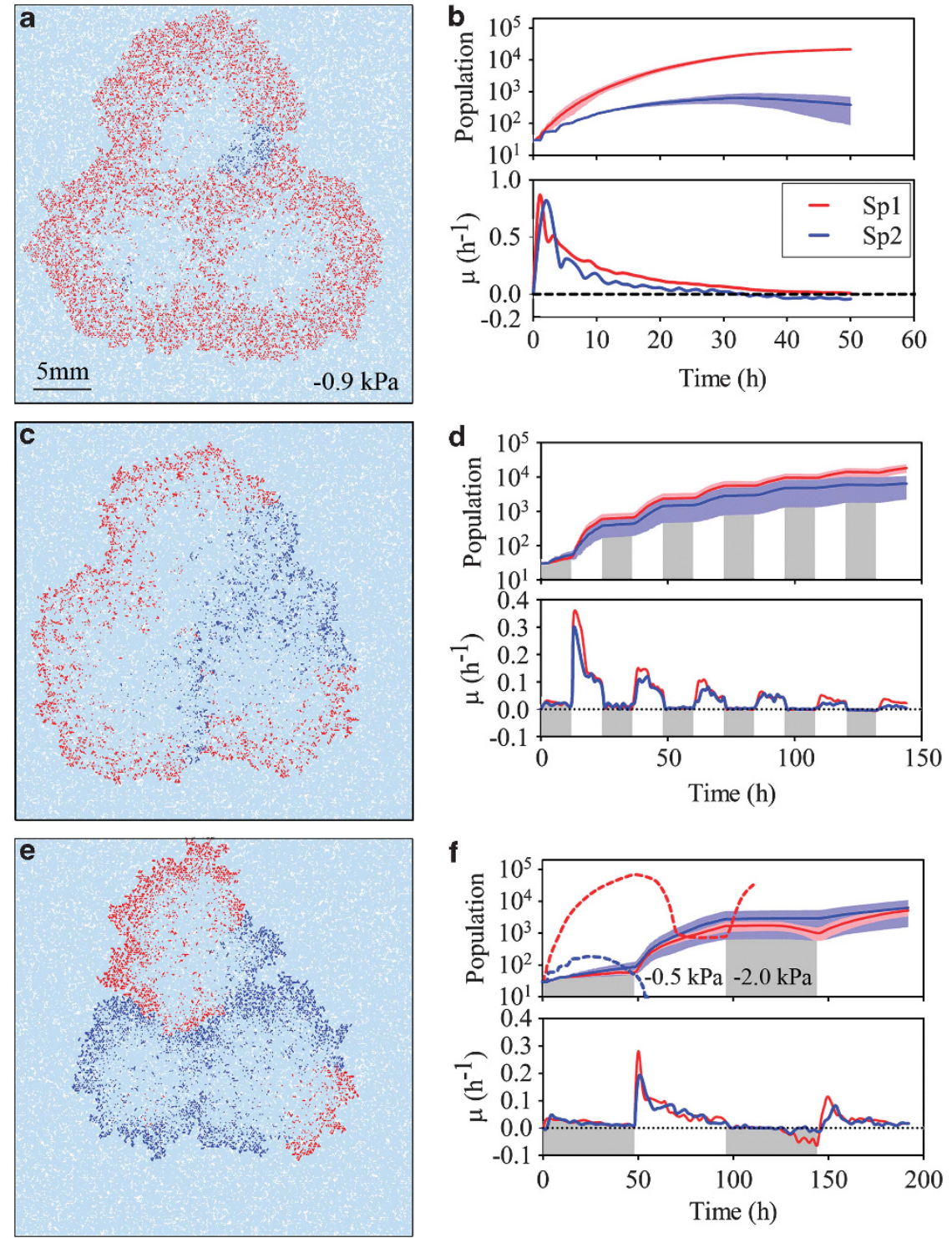

Figure 4 Bacterial growth patterns and population dynamics under different hydration cycles. (a) Simulated bacterial growth patterns, and (b) population growth and specific growth rates under median hydration conditions; and (c) and (e) growth patterns; and (d) and (f) population growth and specific growth rates under short- and long-term dynamic hydration conditions, respectively (dashed curve in (f) illustrates population growth dynamics under hydration cycles started with wet event). Red and blue spots (lines) represent Sp1 and Sp2, respectively. Shaded areas in (b), (d) and (f) represent 1 s.d. of three replicates. Shaded columns in (d) and (f) mark dry episodes of matric potential value of $-2.0 \mathrm{kPa}$.

for all dynamic hydration scenarios (Figures $4 \mathrm{~d}$ and f), consistent with experimental observations (Pesaro et al., 2004; Iovieno and Bååth, 2008; Bapiri et al., 2010). At the end of wet periods (96 h after inoculation for long hydration cycle) nearly $90 \%$ of active cells inhabited domains with relatively large aqueous clusters supported by large water-filled channels. In contrast, most of the surviving bacterial populations were confined to relatively small (and deep) water-filled channels at the end of dry periods (144 h after inoculation for long hydration cycle) (Figure 5). Not surprising, he Sp1 experienced a disproportionally larger reduction in population size due to hydration fluctuations than the reduction in total population size (Figures $5 \mathrm{a}$ and b).
Simulations showed a significant increase in mean relative fitness $(R F)$ of $\mathrm{Sp} 2$ relative to $\mathrm{Sp} 1$ with $R F$ values of 0.35 and 1.20 for short and long dry intervals, respectively, as compared with $R F=0.02$ for static median hydration conditions. These changes reflect a transition from dominance by a superior species to coexistence of the two competing species, a change attributed solely to hydration dynamics (within the same roughness or pore spaces, Figure 4), in agreement with limited experimental observations (McLean and Huhta, 2000; Pesaro et al., 2004). Remarkably, a change in the sequence of hydration dynamics (starting with a wet period) significantly altered the bacterial competition picture, as shown in Figure 4f, resulting in 

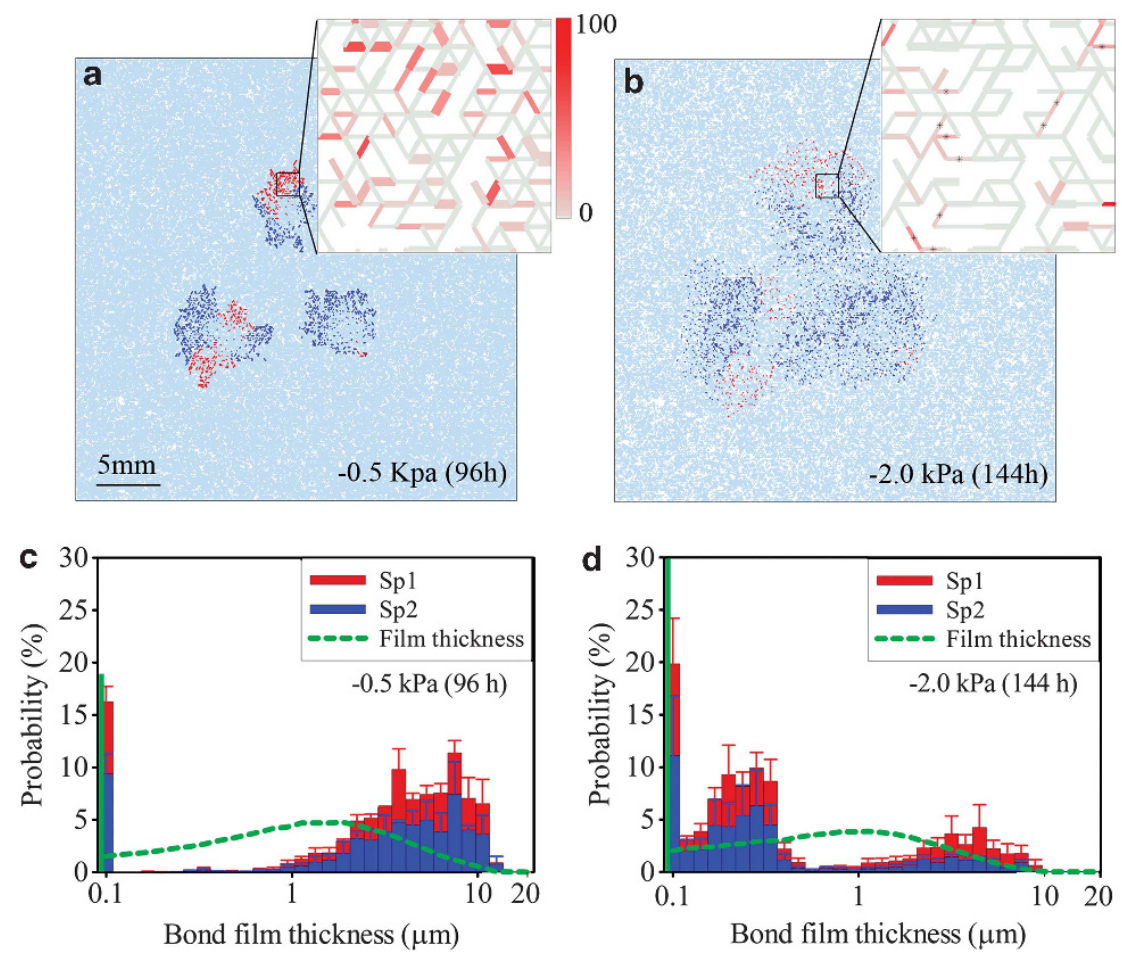

Figure 5 Bacterial growth patterns and population distributions under different episode of long hydration cycles. Simulated bacterial growth patterns at (a) $96 \mathrm{~h}$ (wet) and (b) $144 \mathrm{~h}$ (dry) after inoculations, with zoom-in images showing population densities (star symbols in enlarged inset of (b) mark channels/bonds with aqueous film thickness smaller than cell size); and population spatial distributions (mean \pm s.e.m., $n=3$, marked by the heights of red and blue columns for Sp1 and Sp2, respectively) and aqueous film thickness distributions under (c) wet and (d) dry episodes, respectively. Red and blue spots in (a) and (b) represent individual cells of Sp1 and Sp2. Solid green lines in (c) and (d) mark film thickness $<0.1 \mu \mathrm{m}$.

competitive exclusion of the inferior populations similar to that for static wet scenarios (Figures 2a and d).

\section{Discussion}

The ecological role of hydration dynamics on bacterial growth, community structure and potential influences on species coexistence have been studied in various systems (Chesson, 2000; Ben-Jacob, 2003; Hibbing et al., 2010). The new aspect of this study is in providing some of the first and direct insights into how hydration conditions and associated spatiotemporal variations affect bacterial coexistence and dynamics of community structure. The ease by which aquatic niches become fragmented and disconnected (under dry conditions) accentuates localized growth patterns with temporal sheltering for less competitive species conferring resistance against encroachment by competitors.

Suppression of population growth under dry conditions was attributed primarily to changes in aqueous-phase configuration that affect both motion and diffusion pathways, thereby limiting nutrient fluxes and interception for the competing populations and induce similar (diffusion limited) specific growth rates (Treves et al., 2003; Zhou et al., 2004;
Iovieno and Bååth, 2008). Such mechanism of coexistence promotion by limited diffusive fluxes and aqueous-phase fragmentation is consistent with limited available observations (Zhou et al., 2002; Treves et al., 2003; Ponciano et al., 2009). For example, Zhou et al. (2002) found two to three orders of magnitude higher microbial diversity in unsaturated surface soils than in saturated soils. In addition, restricted bacterial motility within thin aqueous films (dry conditions) limited dispersion distances and reinforced the critical role of localized diffusion pathways on chances of survival (Maennik et al., 2009; Dechesne et al., 2010; Wang and Or, 2010). The experimental results of Dechesne et al. (2008) reveal that for a small drop in matric potential of rough ceramic surfaces (from -0.5 to $-3.6 \mathrm{kPa}$ ) colony expansion rates for motile bacteria dropped by 60 times. These factors contributed to the formation of nearly stationary growth patterns and nutrient-depleted regions that could not be traversed by competitive species (Long and Or, 2005), hence emergence of balanced population competition, and gradually giving rise to coexistence. Under wet conditions $(-0.5 \mathrm{kPa})$, the multitude of hydraulic connections among bacterial habitats maintained pathways for motile bacteria, as well as supported high diffusion links for constant nutrient supply to expanding population 
fronts (Treves et al., 2003; Zhou et al., 2004). These relatively saturated and nutrient-rich simulated scenarios provided Sp1 with a competitive advantage (Treves et al., 2003; Hibbing et al., 2010) that enabled it to expand faster and gradually enclose Sp2's enclaves, thereby intercepting larger fraction of nutrients and tipping competition balance, resulting in competitive exclusion of Sp2. These results are consistent with the classical coexistence theories of niche stabilization and fitness equivalence (Adler et al., 2007), whereby favorable growth environments (high nutrient fluxes, well connected and large aquatic habitats supporting significant cell motion, which are characteristics of wet surfaces) support expression of competitive advantage and lead to the exclusion of inferior species. These often speculated by rarely quantified mechanisms of physicochemical constraints restrict motility and nutrient fluxes within the fragmented aqueous phase, giving rise to fitness equivalence among competing bacterial species (gradual loss of niche difference) and coexistence (Zhou et al., 2002; Treves et al., 2003; Dechesne et al., 2008).

The disproportional sensitivity of densely populated regions to hydration dynamics (drying and wetting) acts to reset population imbalances. These simulation results are in agreement with experimental observations in which drying-rewetting conditions considerably increase bacterial diversity as compared with communities in unstressed and initially wet and fertile soils (Fierer and Schimel, 2002; Fierer et al., 2003). Although physiological traits allowed superior species to establish abundant presence in relatively larger and connected aqueous regions/pores (capitalizing on nutrient supply capacity) within wetting episodes; at the onset of dry periods, nutrient demand required in regions inhabited by large population density cannot be met by the new aqueous-based diffusion field, thereby resulting in a disproportionally large population decay (larger than would be expected for an equivalent reduction in mean flux) (Pesaro et al., 2004; Iovieno and Bååth, 2008; Bapiri et al., 2010). The situation for sparsely distributed 'rural' populations inhabiting harsher domains is different, and simulation results show that a large fraction of the population in these regions survived throughout dry episodes. Moreover, considering motility limitations induced by thinning films (Maennik et al., 2009; Dechesne et al., 2010; Wang and Or, 2010), these sparse and isolated populations are likely to exist in a stationary and sessile form (absent dispersion, expansion or mixing). Consequently, the unsymmetrical decline in population size during drying episodes reinstates a balance in population sizes of the competing species, and contributes to an establishment of nearly stationary coexistence patterns by resetting the ecological clock.

In addition, spatial preferences for dry and wet periods exhibited by bacterial population occupancy are reflections of the complex diffusion field, local nutrient carrying capacity and formation of resilient fraction of the population (less sensitive to hydration perturbations). These factors provide mechanistic explanation for experimental observations that soil drying - rewetting treatments may alter bacterial population dynamics and community structures (Fierer and Schimel, 2002; Fierer et al., 2003; Iovieno and Bååth, 2008). Hence, dynamic hydration conditions temporally increase niche complexity in which location (or diffusion pathway) resilience may affect bacterial population response and even compensate for physiological traits, and thus promote coexistence.

For the rare episodes of prolonged wetting in natural soils, the rapidly expanding population of competitive species may ultimately sweep through the domain including previously sheltered habitats, and lead to competitive exclusion of inferior species as predicted by the classical competition exclusion principles (Hutchinson, 1961). In practice, the ephemeral and very limited time window, when soils are subjected to wet conditions (a few hours per year), reinforces the generality of the physicochemical constraints imposed on nutrient diffusive flux heterogeneity and on motility. Thus sessile life form is expected to dominate soil bacterial life accentuating the ecological consequences of dynamic hydration conditions on bacterial population interactions and community structure across most soil types and climatic regions. The restricted duration of wet events in natural soil also imply severe constraints on distances traversed by motile bacteria into neighboring habitats, which are expected to be limited to a few pores even under favorable conditions (Soby and Bergman, 1983; Dechesne et al., 2010), preventing successful invasions, and thus competitive exclusion of weaker species. Although other agents such as roots and hypha, or concentrated flows in cracks and macropores may contribute to bacterial dispersion over large distances even in relatively dry soils, these would have limited influence along hot spots but are unlikely to alter structured residence of competing populations (Kohlmeier et al., 2005).

Time-averaged nutrient fluxes may be useful for estimations of total population sizes and carrying capacity of soil volumes (Drenovsky et al., 2004; Long and Or, 2007, 2009; Dechesne et al., 2008; Chesson, 2011); however, such averaging would not capture in local interactions discussed above that ultimately shape population dynamics and diversity. For example, inferences based on the average fitness of a species would predict competitive exclusion of weaker species within a bacterial habitat (Carrero-Colón et al., 2006; Chesson, 2011). The results revealed that highly localized, temporally variable and individual-level bacterial interactions, rather than median global values for a species, determine the dynamics and patterns of interacting bacterial populations in systems mimicking natural soils, consistent with organization principles for 
macroscale biological systems (Camazine et al., 2001; Karsenti, 2008). In addition, results show distinct effects of hydration sequences on bacterial coexistence indicative of sensitivity of responses of bacterial populations to initial conditions, which may cast some doubts on inferences based on steady state traditional population models, for example, Lotka - Volterra model (Wangersky, 1978) and niche theories (Adler et al., 2007) to describe complex soil bacterial dynamics.

\section{Conflict of Interest}

The authors declare no conflict of interest.

\section{Acknowledgements}

We acknowledge the financial support of the Swiss National Science Foundation (200021-113442 and 200020-132154-1).

\section{References}

Adler PB, HilleRisLambers J, Levine JM. (2007). A niche for neutrality. Ecol Lett 10: 95-104.

Banavar JR, Maritan A. (2009). Towards a theory of biodiversity. Nature 460: 334-335.

Bapiri A, Bååth E, Rousk J. (2010). Drying-rewetting cycles affect fungal and bacterial growth differently in an arable soil. Microb Ecol 60: 419-428.

Barton JW, Ford RM. (1997). Mathematical model for characterization of bacterial migration through sand cores. Biotechnol Bioeng 53: 487-496.

Berkowitz B, Ewing RP. (1998). Percolation theory and network modeling applications in soil physics. Surv Geophys 19: 23-72.

Ben-Jacob E. (2003). Bacterial self-organization: coenhancement of complexification and adaptability in a dynamic environment. Phil Trans $R$ Soc $A$ 361: 1283-1312.

Blunt MJ. (2001). Flow in porous media: pore-network models and multiphase flow. Curr Opin Colloid Interface Sci 6: 197-207.

Camazine S, Deneubourg J, Franks NR, Sneyd J, Theraulaz G, Bonabeau E. (2001). Self-organization in biological systems. Princeton University Press: Princeton, NJ, pp. 29-46.

Carrero-Colón M, Nakatsu CH, Konopka A. (2006). Microbial community dynamics in nutrient-pulsed chemostats. FEMS Microbiol Ecol 57: 1-8.

Chang WS, Halverson LJ. (2003). Reduced water availability influences the dynamics, development, and ultrastructural properties of Pseudomonas putida biofilms. J Bacteriol 185: 6199-6204.

Chen J, Jin Y. (2011). Motility of Pseudomonas aeruginosa in saturated granular media as affected by chemoattractant. J Contam Hydrol 126: 113-120.

Chesson P. (2000). Mechanisms of maintenance of species diversity. Annu Rev Ecol Evol Syst 31: 343-366.

Chesson P. (2011). Ecological niches and diversity maintenance. In: Pavlinov IY (ed). Research in biodiversity-models and applications. InTech: Rijeka, pp. 43-60.
Crawford JW, Harris JA, Ritz K, Young IM. (2005). Towards an evolutionary ecology of life in soil. Trends Ecol Evol 20: 81-87.

Curtis TP, Sloan WT. (2004). Prokaryotic diversity and its limits: microbial community structure in nature and implications for microbial ecology. Curr Opin Microbiol 7: 221-226.

Darnton NC, Berg HC. (2008). Bacterial flagella are firmly anchored. J Bacteriol 190: 8223-8224.

Darrah PR. (1991). Measuring the diffusion coefficient of rhizosphere exudates in soil. I. The diffusion of nonsorbing compounds. J Soil Sci 42: 413-420.

Dechesne A, Or D, Gulez G, Smets BF. (2008). The porous surface model, a novel experimental system for online quantitative observation of microbial processes under unsaturated conditions. Appl Environ Microbiol 74: 5195-5200.

Dechesne A, Wang G, Güleza G, Or D, Smets BF. (2010). Hydration-controlled bacterial motility and dispersal on surfaces. Proc Natl Acad Sci USA 107: 14369-14372.

Dens EJ, Van Impe JF. (2000). On the importance of taking space into account when modeling microbial competition in structured food products. Math Comput Simulat 53: 443-338.

Drenovsky RE, Vo D, Graham KJ, Scow KM. (2004). Soil water content and organic carbon availability are major determinants of soil microbial community composition. Microb Ecol 48: 424-430.

Elena SF, Lenski RE. (2003). Evolution experiments with microorganisms: the dynamics and genetic bases of adaptation. Nat Rev Genet 4: 457-469.

Fenchel T. (2002). Microbial behavior in a heterogeneous world. Science 296: 1068-1071.

Fierer N, Jackson R. (2006). The diversity and biogeography of soil bacterial communities. Proc Natl Acad Sci USA 103: 626-631.

Fierer N, Lennon JT. (2011). The generation and maintenance of diversity in microbial communities. Am J Bot 98: 439-448.

Fierer N, Schimel JP. (2002). Effects of drying-rewetting frequency on soil carbon and nitrogen transformations. Soil Biol Biochem 34: 777-787.

Fierer N, Schimel JP, Holden PA. (2003). Influence of drying-rewetting frequency on soil bacterial community structure. Microb Ecol 45: 63-71.

Gordon H, Haygarth PM, Bardgett RD. (2008). Drying and rewetting effects on soil microbial community composition and nutrient leaching. Soil Biol Biochem 40: 302-311.

Hibbing ME, Fuqua C, Parsek MR, Peterson SB. (2010). Bacterial competition: surviving and thriving in the microbial jungle. Nat Rev Microbiol 8: 15-25.

Hutchinson GE. (1961). The paradox of the plankton. Am Nat 95: 137-145.

Iovieno P, Bååth E. (2008). Effect of drying and rewetting on bacterial growth rates in soil. FEMS Microbiol Ecol 65: $400-407$.

Karsenti E. (2008). Self-organization in cell biology: a brief history. Nat Rev Mol Cell Biol 9: 255-262.

Kreft JU, Booth G, Wimpenny JWT. (1998). BacSim, a simulator for individual-based modelling of bacterial colony growth. Microbiology 144: 3275-3287.

Kohlmeier S, Smits THM, Ford RM, Keel C, Harms H, Wick LY. (2005). Taking the fungal highway: mobilization of pollutant-degrading bacteria by fungi. Environ Sci Technol 39: 4640-4646. 
Long T, Or D. (2005). Aquatic habitats and diffusion constraints affecting microbial coexistence in unsaturated porous media. Water Resour Res 41: W08408.

Long T, Or D. (2007). Microbial growth on partially saturated rough surfaces: simulations in idealized roughness networks. Water Resour Res 43: W02409.

Long T, Or D. (2009). Dynamics of microbial growth and coexistence on variably saturated rough surfaces. Microb Ecol 58: 262-275.

Maennik J, Driessen R, Galajda P, Keymer JE, Dekker C. (2009). Bacterial growth and motility in sub-micron constrictions. Proc Natl Acad Sci USA 106: 14861-14866.

McLean MA, Huhta V. (2000). Temporal and spatial fluctuation in moisture affect humus microfungal community structure in microcosms. Biol Fertil Soils 32: 114-119.

Mitchell JG, Kogure K. (2006). Bacterial motility: links to the environment and a driving force for microbial physics. FEMS Microbiol Ecol 55: 3-16.

Moldrup P, Olesen T, Komatsu T, Yoshikawa S, Schjønning P, Rolston DE. (2003). Modeling diffusion and reaction in soils: X. A unifying model for solute and gas diffusivity in unsaturated soil. Soil Sci 168: 321-337.

O’Donnell AG, Young IM, Rushton SP, Shirley MD, Crawford JD. (2007). Visualization, modelling and prediction in soil microbiology. Nat Rev Microbiol 5: 689-699.

Or D, Smets BF, Wraith JM, Dechesne A, Friedman SP. (2007). Physical constraints affecting bacterial habitats and activity in unsaturated porous media - a review. Adv Water Resour 30: 1505-1527.

Pesaro M, Nicollier G, Zeyer J, Widmer F. (2004). Impact of soil drying-rewetting stress on microbial communities and activities and on degradation of two crop protection products. Appl Environ Microbiol 70: 2577-2587.

Ponciano JM, La HJ, Joyce P, Forney LJ. (2009). Evolution of diversity in spatially structured Escherichia coli populations. Appl Environ Microbiol 75: 6047-6054.

Prosser JI, Bohannan BJM, Curtis TP, Ellis RJ, Firestone MK, Freckleton RP et al. (2007). The role of ecological theory in microbial ecology. Nature 5: 384-392.

Reichenbach T, Mobilia M, Frey E. (2007). Mobility promotes and jeopardizes biodiversity in rock-paperscissors games. Nature 448: 1046-1049.
Saragosti J, Calvez V, Bournaveas N, Perthame B, Buguin A, Silberzan P. (2011). Directional persistence of chemotactic bacteria in a traveling concentration wave. Proc Natl Acad Sci USA 108: 16235-16240.

Soby S, Bergman K. (1983). Motility and chemotaxis of Rhizobium meliloti in soil. Appl Environ Microbiol 46: 995-998.

Stotzky G. (1997). Soil as an environment for microbial life. In: van Elsas JD, Trevors JT, Wellington EMH (eds). Modern Soil Microbiology. Marcel: New York, pp 1-20.

Treves DS, Xia BC, Zhou JZ, Tie JM. (2003). A twospecies test of the hypothesis that spatial isolation influences microbial diversity in soil. Microb Ecol 45: 20-28.

Torsvik V, Ovreas L. (2002). Microbial diversity and function in soil: from genes to ecosystems. Curr Opin Microbiol 5: 240-245.

Torsvik V, Ovreas L. (2008). Microbial diversity, life strategies, and adaptation to life in extreme soils. In: Dion P, Nautiyal CS (eds). Microbiology of Extreme Soils. Springer: Berlin, Heidelberg, pp 15-43.

Tsyganov MA, Ivanitsky GR. (2006). Solitonlike and nonsoliton models of interaction of taxis waves. Biophysics 51: 887-891.

Vos M, Velicer GJ. (2008). Natural variation of gliding motility in a centimeter-scale population of Myхососcus xanthus. FEMS Microbiol Ecol 64: 343-350.

Wangersky PJ. (1978). Lotka-Volterra population models. Ann Rev Ecol Evol Syst 9: 189-218.

Wang G, Or D. (2010). Aqueous films limit bacterial cell motility and colony expansion on partially saturated rough surfaces. Environ Microbiol 12: 1363-1373.

Wilson PDG, Brocklehurst TF, Arino S, Thuault D, Jakobsen M, Lange M et al. (2002). Modelling microbial growth in structured foods: towards a unified approach. Int J Food Microbiol 73: 275-289.

Zhou JZ, Xia BC, Huang HS, Palumbo AV, Tiedje JM. (2004). Microbial diversity and heterogeneity in sandy subsurface soils. Appl Environ Microbiol 70: 1723-1734.

Zhou JZ, Xia BC, Treves DS, Wu LY, Marsh TL, O’Neill RV et al. (2002). Spatial and resource factors influencing high microbial diversity in soil. Appl Environ Microbiol 68: 326-334. 